\title{
LA FAMILIA LAKHOUA, DESCENDIENTES TUNECINOS DE MORISCOS GRANADINOS DE LOS SIGLOS XVII-XVIII, Y SUS ACTIVIDADES EN LA INDUSTRIA DEL BONETE CHECHÍA ${ }^{1}$
}

\author{
Abdel-Hakim Gafsi Slama
}

La familia tunecina de los Lakhoua ${ }^{2}$, de origen andalusí indudable y de gran importancia social en este país, hasta nuestros días, es notable por dos aspectos particulares. Por una parte, porque algunos miembros de esta familia son moriscos que salieron de Granada a mediados del siglo XVIII y no sólo en el siglo XVII, cuando la gran expulsión de los moriscos, ya que está documentada su llegada a Túnez el verano del año 1731, aunque también

1. Quiero expresar mi agradecimiento al profesor Míkel de Epalza, catedrático de la Universidad de Alicante, por su ayuda en forma de recomendaciones, sugerencias y correcciones.

2. Forma afrancesada usual, en letras latinas, del nombre árabe al-ijwa, con significado celos hermanos». Sobre el origen de este apellido, al llegar parte de esta familia a Túnez en el siglo XVIII, ver M. de EPALZA, «Nuevos documentos sobre descendientes de los moriscos en Túnez en el siglo XVIII», Studia historica et philologica in honorem M. Batllori, Roma, 1984, pp. 213-214 [traducido al árabe, en la revista tunecina Al-Maŷlla at-Tärījiyya al-Magribiyya, y al francés: «Nouveaux documents sur les Andalous en Tunisie au début du XVIIIe siècle», Revue d'Histoire Maghrébine, 17-18, Túnez, 1980, pp. 79-108, y en S.M. ZBISS; A.-H. GAFSI; M. Boughanmi y M. de Epalza, Études sur les Morisques Andalous, Túnez (Ed. Institut National d'Archéologie et d'Art), 1983, pp. 67-70, y en este trabajo, infra. Sobre apellidos de descendientes de moriscos en Tunicia, ver especialmente Nabila ZBISS, a«L'onomastique espagnole en Tunisie», Sharq al-Ándalus. Estudios Árabes, 7 Alicante, 1990, pp. 215-219, y M. de EPALZA y A.-H. GAFSI-SLAMA, «Léxico y onomástica hispánicos de los moriscos conservados en Tunicia», en Actas del VII Simposio Internacional de Mudejarismo, Teruel, Centro de Estudios Mudéjares-IET, 1999, pp. 633-641, y libro en preparación, de los mismos autores, Léxico y onomástica hispánicos de los moriscos, conservados en Tunicia. En este artículo se utilizará la transcripción moderna, afrancesada, de Lakhoua. La del siglo XVIII, en castellano, era La Joa, según el diario de Francisco Ximénez, a 26 de julio de 1731. Ver «Nuevos documentos...». 
están documentados unos ricos Lakhoua a fines del siglo anterior ${ }^{3}$. Pero, por otra parte, ya habían adquirido por esas fechas -los de la familia granadina $u$ otros andalusíes con el mismo apellido- una gran importancia en la industria artesanal del bonete "chechía", el principal objeto de exportación de la Regencia de Túnez en el siglo XVIII, que seguirá siendo importante en el XIX y se mantiene, ya en forma residual, hasta nuestros días ${ }^{4}$.

\section{ORIGEN DE LOS LAKHOUA GRANADINOS Y SU INSTALACIÓN EN TUNICIA EN EL SIGLO XVIII}

Un curioso episodio, en el primer tercio del siglo XVIII (entre 1727 y 1732), va a renovar la situación de unos cripto-musulmanes que se habían mantenido en secreto en la sociedad española granadina ${ }^{5}$. Descubiertos, por

3. Varios autores afirman, sin aportar pruebas concretas, que los Lakhoua emigraron cuando la gran expulsión de principios del XVII: "Ils [los Lakhoua] auraient quitté l'Espagne au moment du grand exode des Andalous chassés par Philippe III en 1609" (Jacques ReVault, Palais et demeures de Tunis (XVIII el XIXe s.), Paris, C.N.R.S., 1983, p. 150) y “Arrivés à Tunis au XVIIe siècle, aprés un séjour au Maroc, les Lakhoua entrent probablement dans la profession des shawwashi-s fabricants et marchands exportateurs et y demeurèrent fidèles jusqu'à nos jours” (M.A. BEN ACHOUR, «Les Aylat du corpus: notes historiques», en Hasab wa nasab. Parenté, alliance et patrimoine en Tunisie, París, C.N.R.S. 1992, p. 130. Sí aparecerán algunos Lakhoua en Túnez en la documentación de fines del XVII y principios del XVIII presentada por S. Boubaker (ver más adelante), antes de la llegada de los Lakhoua granadinos de 1731, según documento publicado por M. de EPALZA, «Nuevos documentos sobre descendientes de moriscos en Túnez en el siglo XVIII», Studia historica el philologica in honorem M. Batllori, Roma, 1984, 195-228, reproducido en traducciones francesas y árabe, en Túnez.

4. El principal estudio sobre la estructura de esta artesanía sigue siendo el de la antropóloga tunecina Sophie FERCHIOU, Technique et société: la fabrication de la chéchia en Tunisie, Paris, Institut d'Ethnologie, 1971, que no trata más que de pasada de sus aspectos históricos, fundamentales en cambio en el artículo de Robert RICARD, «L'Espagne el la fabrication des bonnets tunisiens: à propos d'un texto du XVIIIe siécle», Revue Africaine, C, Argel, 1956, pp, 426-437. Ver también artículo, más tradicional y con aspectos históricos, de Muhammad ANNABI, «La chéchia tunisienne», en M. de Epalza y R. PetiT, Recueil d'études sur les Morisques Andalous en Tunisie, Madrid, Ministerio de Asuntos Exteriores, 1974, pp. 304-307. Sobre el vocabulario en español de esta artesanía, conservado hasta nuestros días, ver infra la encuesta de Paul Teyssier. Visión general de la importancia de los moriscos y sus descendientes en esta industria artesanal en Abdel-Hakim EL GAFSI, «Al-mūriškūs al-andalusiyyūn wa-tiȳārat aș-șūf [Los moriscos andalusíes y el comercio de la lana]», Les Cahiers de Tunisie, 155-156, Tunis, 1991, pp. 53-85. Sobre la evolución de esta industria en España, después de la gran expulsión de los moriscos en el siglo XVII, ver el texto de Jovellanos de fines del XVIII, apéndice documental de este estudio.

5. Tema ya estudiado por Henry Charles Lee, Julio Caro Baroja y otros, pero ampliamente tratado y documentado por Rafael de LERA GARCÍA, «Cripto-musulmanes ante la Inquisición granadina en el s. XVIII», Hispania Sacra, XXXVI, Madrid, 1984, pp. 1-55. Resumen de sus conclusiones, en francés, en «Survie d'Islam dans la ville de Grenade au debut du XVIIle siècle», Revue d'Histoire Maghrébine, 43-44, Zaghouan, 1986, pp. 59-82. 
denuncias mutuas ante la Inquisición, se ven juzgados con bastante lenidad por los tribunales inquisitoriales. Se conserva un curioso texto de la Inquisición que resume la doctrina y las prácticas por las que fueron condenados finalmente a penas leves y que termina con los párrafos siguientes:

\begin{abstract}
"Advirtiendo que era tanto el conjunto de disparates que observaban tan sin método, que ninguno de dichos reos ha savido dar razón de los ayunos, rezos y demás ceremonias, pues no se ha verificado que dos estén conformes en guardar una misma cosa, observando unos unas ceremonias y otros otras inmediatamente opuestos, sin tener uniformidad en cosa alguna de su secta, de la qual regularmente eran los maestros las mugeres, quienes tenían el cuidado de instruir a sus hijos, parientes o criados, desde la hedad de ocho años hasta la de doce o catorce, valiéndose del medio de decirles que eran descendientes de moros de los que se quedaron en la conquista de este reyno, y que ellos y sus mayores avían seguido su secta de Mahoma, que era la más segura para salvarse, y como era esta enseñanza en tan tierna hedad y a persuasión de las madres, la admitían, y quando llegaban a tener discreción, aunque muchos conocían ser verdaderamente herrores los que seguían, por no descubrir a sus padres y parientes, se mantenían en ellos; incurrían también en la maldad de enseñar a sus hijos cuando llegaban a tener hedad proporcionada para ello, pero todos han estado fáciles en confesar y han dado buenas muestras de su arrepentimiento, y que no volverán a reincidir en su secta. Dios Nuestro Señor les dé perseverancia en estos propósitos. Amen”.6.
\end{abstract}

Por ese mismo texto, se ve la línea de defensa que escogieron esos fieles musulmanes, ante los tribunales inquisitoriales. De hecho, fueron condenados a penas leves, pero algunos de los personajes más importantes fueron desterrados de Granada, no fuera de España, sino a otras regiones del país y por un tiempo determinado ${ }^{7}$.

Algunos de esos adinerados granadinos se mantenían, en realidad, bastante fieles al Islam. Instalados en Barcelona, lograron emigrar a países musulmanes, concretamente a Esmirna, puerto de Anatolia occidental, bajo dominio del Imperio Otomano ${ }^{8}$. De ahí intentan y logran instalarse en la

6. María Soledad CARRASCo URGOITI y M. de EPALZA, «El manuscrito "Errores de los moriscos de Granada" (Un núcleo criptomusulmán en el primer tercio del siglo XVIII)», Fontes Rerum Balearium, III, Palma de Mallorca, 1979-1980, pp. 235-247 (p. 247).

7. Es posible que pesara en la relativa levedad de estas condenas inquisitoriales una reflexión histórica sobre la responsabilidad de la expulsión a países musulmanes, que propició que se reforzaran militar y económicamente esos países y que "se perdieran" las almas de esos expulsados y de sus descendientes, al criarse de nuevo en la religión musulmana, con la práctica imposibilidad de vivir y morir en el cristianismo, según la visión teológica de las autoridades católicas españolas.

8. Ver nombres y detalles en el estudio de R. de VERA GARCÍA, «Cripto-musulmanes...». Sobre los moriscos y su instalación en el Imperio Otomano, ver estudio y bibliografía recientes de M. de EPALZA, «Instalación de moriscos en Anatolia (documento Temimi, de 1613)», Sharq al-Andalus. Estudios Mudéjares y Moriscos, 13, Teruel-Alicante, 1996, pp. 145-157. 
Regencia de Túnez, según un texto del diario en el que anotaba los acontecimientos de cada día el religioso español fray Francisco Ximénez, Director del Hospital español de los cristianos, en la capital tunecina. Escribe Ximénez, a fecha del 27 de julio de 1731:

"Ha escrito desde Esmirna a Cherife Castelli un cierto Moza La Ja que dice ser descendiente de los Albencerrajes, natural de Granada, alcaide de la torre del Aceitunero y puerta de la Taxalanza, el cual fue por la inquisición de Granada castigado por morisco a cuatro años de destierro y se ha pasado con sus hermanos y hermanas a Esmirna. De allí pretende venir a vivir a esta ciudad. Habrá cuatro años que fue castigado"”.

El nombre árabe del jefe de este grupo de inmigrantes permite hacer el lazo entre los granadinos y sus actuales descendientes tunecinos: Muza (Musa, Moisés) La Joa (al-ijwa, que significa "los hermanos", precisamente "sus hermanos y hermanas" del texto español, que les quedó como apellido).

Pero el texto de Francisco Ximénez añade más: informa que los granadinos refugiados en el Imperio Otomano se dirigen a Cherife Castelli, que era una gran personalidad andalusí descendiente de moriscos inmigrantes del XVII, riquísimo comerciante y compatriota del Jaznadar o ministro de finanzas del Bey o gobernador de la provincia del Imperio Otomano o regencia muy autónoma de Túnez. De ambos magnates andalusíes de Tunicia, descendientes de moriscos, trata ampliamente Francisco Ximénez en su diario ${ }^{10}$.

Esta relación inicial entre los Castelli y los también adinerados Lakhoua explicaría la importancia que han tenido éstos en la industria artesanal de la chechía o bonete tunecino. Los Lakhoua aportaban dinero y lo invirtieron en esa fabricación, que era también uno de los tres pilares de la riqueza de los Castelli, en la Tunicia del siglo XVIII, con el corso y el comercio de esclavos ${ }^{11}$.

9. Ver M. de EPALZA, «Nuevos documentos...», pp. 213-214. Noticia de este texto en S. CARRASCO URGOITI y M. de EPALZA, op. cit., p. 236, y estudio de su contexto histórico general en M. de EPALZA, «Estructuras de acogida de los moriscos emigrantes de España en el Magreb (siglos XIII al XVIII)», Alternativas. Cuadernos de Trabajo Social, 4, Alicante, 1996, pp. 35-58 (especialmente pp. 50-53).

10. Ver M. de EPALZA, «Nuevos documentos...», especialmente pp. 197-208.

11. Ver análisis documentado de esta estrategia económica en A.-H. GAFSI, «Une famille de monétaires sous les Husseinites: les Kastalli», Monnaies Tunisiennes depuis l'époque punique jusqu'à nos jours, Tunis, Ed. Banque de Tunisie, 1993, pp. 23-40. Sobre la riqueza de algunos moriscos y descendientes de moriscos, en el Magreb, ver Denise BRAHIMI, «Quelques jugements sur les maures andalous dans les régences turques au XVVIIe siècle», en M. de EPALZA y R. PETIT, op. cit., pp. 135-149. 


\section{LOS MORISCOS Y LA CHECHÍA O BONETE TUNECINO EN LOS SIGLOS XVII Y XVIII}

Ya desde la instalación en Túnez y su región de los moriscos expulsados de España, a principios del siglo XVII, se manifiesta un renacer de la fabricación de la chechía o bonete tunecino, en relación con los inmigrantes moriscos y sus descendientes, como aparece en la documentación comercial del Consulado de Francia en esa capital, a lo largo de ese siglo ${ }^{12}$. Allí aparecen los andalusíes Alli Cherif y Mehemet Facar, "maîstre bonnetiers de Tunis", que presentan una queja por la mala calidad del tinte bermellón que les ha vendido un mercader genovés de Marsella; ese tinte se importaba generalmente de España, precisamente por Génova o Marsella ${ }^{13}$. Al final del siglo, el jefe o Shaij de los andalusíes de Túnez, Sidi Hamet, también figura como fabricante y comerciante de chechía ${ }^{14}$. Pero también aparece documentación de comercio internacional de chechías a mediados del siglo XVII: los mercaderes andalusíes Isuf Sanmar y Sta Mammet l'Eschiabo declaran estar muy satisfechos del patrón francés del navío San Giuseppe, que les ha llevado a Génova un cargamento de jabón y de gorros árabes, para venderlo al mejor precio ${ }^{15}$, y, ya pocos años después de la expulsión de 1609-1614, el jefe de los andalusíes Juan Pérez da préstamos con garantías de lana de chechía, en 1616, al igual que Alonso de Cuevas o Cuebas, llamado en morisco Mahamet, ese mismo año ${ }^{16}$.

En el siglo XVIII, la documentación sobre la chechía es ya muy abundante y ha dado origen a numerosos estudios, recogidos en la bibliografía general ya mencionada.

Ya en 1727/1140 h., una lista de 22 tiendas, donde se trabajaban y se vendían las chechías en el zoco particular que había hecho construir el Bey o soberano de Túnez junto a su palacio, nos da numerosos nombres de familias andalusíes muy conocidas, algunas con nombres hispanos (Castelli, Huesca, Lorca, Palma, Córdoba, Chiquito, Esclavo,...) y cuatro tiendas perte-

12. Documentación estudiada, en relación con los moriscos o andalusíes del país, en M. de EPALZA, «Moriscos y andalusíes en Túnez durante el siglo XVII», Al-Andalus, XXXIV/2, Madrid, 1969, pp. 247-327 [traducido al francés, con nuevos índices onomásticos, en M. de EpALZA y R. PETIT, op. cit., pp. 150-186].

13. Ver M. de EPALZA, «Moriscos y andalusíes...», pp. 272, 279 y 282, con documentación de 1686.

14. Ibídem, pp. 272, 279 y 292, con documentación de 1697, relacionada con comercio de bonetes con Francia.

15. Ibídem, p. 274. Es documentación de 1655.

16. Ibídem, pp. 272 у 275. 
necientes a miembros de la familia Lakhoua: Muhámmad Lakhoua, Alí Lakhoua, Yúsuf Lakhoua y Al-Hach Muhámmad Lakhoua ${ }^{17}$.

Esta industria artesanal había llegado a ser muy importante y la primera materia de exportación de la Regencia. Llegó a ocupar una abundante mano de obra, tanto en la capital como en varios pueblos rurales de Tunicia (Batán, El Alia, Zaghouan...), especializados cada uno de ellos en etapas diferentes de la producción de la chechía. Lucette Valensi calcula que ocuparían entre 15.000 y 80.000 personas $^{18}$.

El papel de los andalusíes en la chechía se puede apreciar también por el testimonio del ya mencionado Francisco Ximénez, religioso trinitario y director del Hospital cristiano de Túnez, entre 1720 y $1735^{19}$. Estos textos son muy importantes, porque en su minucioso diario, durante esos quince años, aparecen incidentalmente los "birretes", bonetes o chechías, relacionados con los más ricos y poderosos comerciantes tunecinos, con gran poder político y económico en la corte del primer soberano de la dinastía Husainí, dinastía que gobernaría Tunicia con autonomía, aunque bajo la soberanía nominal del Imperio Otomano, más de dos siglos y medio (1700-1956).

El poderoso primer ministro y ministro de finanzas (jaznadar), el andalusí Mahamut Jasnadal, y su compatriota Cherife Castelli tienen gran parte de su riqueza invertida en esa producción y comercio. En el viaje que hace Ximénez al pueblo de Los Batanes (actual Batán), escribe (en los primeros días de marzo de 1727): "Hay batanes donde se abatanan las birretas coloradas que usan los moros. Son del Bey y los tiene por su cuenta Cherife Castelli, moro originario castellano... Sobre ellos tiene una casa donde fuimos alojados. Y hay también molino de hacer harina... Fuimos al borche de Mahamet, que está una legua de aquí, a las márgenes del mismo [río] Macherda. Aquí hay un puente que tiene $24 \operatorname{arcos,~los~cuatro~últimos~ocupan~}$ molino y batanes para las birretas, que corren también por cuenta de Cherife Castelli. Esta puente la hizo Mahamet Bey... En los postes de los arcos se dilatan fuera del puente. Muchos

17. Ver A.-H. AL-GAFSI, «Naẓra hawla ba‘ḍ al-hịrafiyyīn wa-l-mihniyyīn al-andalusiyyīn wa-latrāk bi-l-aŷāla at-tūnisiyya atnā'a al-qarn at-tāsi' ‘ašar min jilāl hazāinat al-watāa' iq» [«Presentación general de algunos cargos y artesanos andalusíes y turcos en la Regencia de Túnez en el siglo XIX, según documentos archivísticos»], en A. Temimi (ed.), Actes du congrés sur la vie sociale dans les provinces arabes à l'époque ottomane, Zaghouan, Centre d'Études et de Recherches Ottomanes, Morisques, de Documentation et d'Information (C.E.R.O.M.D.I.), 1988, pp. 599-611 (especialmente pp. 605-606).

18. Ver L. VALENSI, «Islam et capitalismo: production et commerce des chéchias en Tunisie et en Franco aux XVIIIe et XIXe siècles», Revue d'Histoire Moderne et Contemporaine, París, 1969, p. 931.

19. Sobre la minuciosidad y objetividad "periodística" de Francisco Ximénez como fuente documental, ver la monografía de M. de EPALZA, «La expedición de Alicante para la toma de Orán (1732) y su seguimiento en Túnez», Sharq al-Andalus. Estudios Árabes, 2, Alicante, 1985, pp. 11-18 [traducción francesa en «Un cas d'opinion publique maghrébine: la prise d'Oran par les Espagnols, vue de Tunis (1732)», Revue d'Histoire Maghrébine, 55-56, Zaghouan, 1989, pp. 5-10]. 
dicen hizo así esta fábrica para hacer allí zugas, para sacar agua, regar la campaña y traer a Túnez. Para hacer esta fábrica derribó los edificios soberbios de los romanos de diversas partes, para traer la piedra con las carretas de los moros andaluces..." ${ }^{20}$. Los batanes del puente y pueblo de El Batán, junto a Tebourba, sobre el río Medjerda, seguirán siendo el centro del proceso de abatanamiento de la lana de las chechías, hasta época moderna ${ }^{21}$.

En muchos otros textos de su diario Francisco Ximénez menciona ricos andalusíes, pero sólo en uno se refiere expresamente a la relación de esos burgueses con las chechías. Cuando el Bey encausa a un grupo de ricos andalusíes, relacionados con una conjura contra el soberano, dice de uno de ellos (diario, en fecha de 17 de julio de 1729): "Han preso, por rebelde al Bey, a Méndez, moro andaluz que era bien rico y tenía oficio de birretero" 22 .

Francisco Ximénez había estado dos años en las ciudades argelinas de $\operatorname{Orán}^{23}$ y de $\operatorname{Argel}^{24}$, pero allí nada dejó escrito en su diario sobre las relaciones de los andalusíes con las industrias artesanales como la chechía. Documentos de mediados del siglo anterior sí que muestran la riqueza de algunos comerciantes de chechía en $\operatorname{Argel}^{25}$.

20. M. de EPALZA, «Nuevos documentos...», p. 207. Francisco Ximénez entiende por "andaluces" los “andalusíes", según la denominación árabe en la Tunicia del s. XVIII.

21. Ver curioso documento de 1909/1327 h., estudiado por A.-H. GAFSI, «Watāà iq ŷadīda ḥawla iqāma "bāțān” ŷadīd li-gasl aš-šăšiyya [Nuevos documentos sobre la construcción de un nuevo batán para la limpieza de las chechías]», Revue des arts et traditions populaires, 8, Túnez, 1984, pp. 5-9. Uno de los ocho miembros del Consejo de los fabricantes de chechía (maŷlis aš-šawā̌siyya) era por entonces (1904) At-Tāhir Lakhoua, de la familia que estudiamos (ibidem, nota 7).

22. M. de EPALZA, «Nuevos documentos...», p. 209.

23. Ver M. de EPALZA y Hedi OuESLATI, «Observaciones de un eclesiástico español de paso por Orán en tiempos de Mustafá Bushlághim» [en árabe], Al-Ma ŷalla at-Tārījiyya al-Magribiyya, 12, Túnez, 1978, pp. 191-203. Ximénez permaneció en Orán sólo unos meses, en 1718, durante el cuarto de siglo aproximadamente (1708-1732) de dominio argelino -bajo el Bey Bushlághim- entre las dos ocupaciones españolas, en un intento de establecer en la ciudad un hospital para los esclavos europeos como había en Argel. No pudo haber en esa ciudad, que era por tanto española en el momento de la expulsión del XVII, ninguna implantación de moriscos durante este período. Sobre el papel de Orín en la expulsión de los moriscos y el cuadro valenciano contemporáneo que representa el paso de los moriscos por Orán, ver M. de EPALZA, «Los moriscos y sus descendientes, después de la expulsión (Después del cuadro del desembarco en Orán)», en José MIRANDA (coord.), La expulsión de los moriscos, Valencia, ed. Fundación BANCAJA, 1998, pp. 41-70.

24. Ver H. OUESLATI, «Argel, según el diario inédito de Francisco Ximénez (1718-1720)», Sharq al-Andalus. Estudios Árabes, 3, Alicante, 1986, pp. 169-181. Ximénez sólo menciona la instalación de descendientes de moriscos en Argel y su región, sin precisar el origen de su riqueza [ver pp. 172 (instalación en Blida), 174 (como grupo particular de la población argelina) y 179-180 ("tagarinos" ricos de origen morisco)].

25. Ver Ferid KHIAIRI, «Une communauté resurgente: les Andalous en Alger de 1570 à 1670», Revue d'Histoire Maghrébine, 69-70, Túnez, 1993, pp. 119-132 [según el testamento de un fabricante de chechías, de 1663]. 
Más tarde, seguramente de vuelta ya a España, Francisco Ximénez escribe un libro sobre la historia del Hospital de los religiosos trinitarios en $T^{u} n z^{26}$. En él da muchas noticias sobre los andalusíes o descendientes de moriscos en Túnez, resumiendo sus informaciones y no dispersándolas, como en el diario ${ }^{27}$. Varias se refieren precisamente al papel de los moriscos y sus descendientes en la industria artesanal de la chechía. Por su importancia de testimonio directo del primer tercio del siglo XVIII, reproducimos estos textos a continuación.

\footnotetext{
"Los moros andaluces [andalusíes] [...] tienen el uso de carros y carretas. Sólo ellos hacen queso y trabajan otras manufacturas que sus ascendientes trajeron de España..."28.
}

En la capital, Túnez, “[...] hay muchos fabricantes de telas de seda, algodón, lino y lana, y de las mejores birretas rojas; muchas de estas manufacturas trajeron los moriscos españoles de España" 29 .

Más adelante, ya especifica la importancia de los moriscos o andalusíes y de su industria artesanal de bonetería o chechía: "Una de las cosas principales que hacen apreciable el Reyno de Túnez es el tráfico y comercio. Esto consiste especialmente en todo lo que sirve a la fábrica de los bonetes rojos, que los turcos y moros traen debajo de sus turbantes. Es muy bella y estimada la fábrica, manera y suerte de los bonetes y se reparten por toda la Berbería y Levante. Antiguamente se los fabricaban los moriscos en España, en Toledo y otras partes, y cuando pasaban al África los redentores Trinitarios, llevaban cantidad de ellos, para trocarlos por cautivos. Después de la expulsión de los moriscos se acabó en España esta fábrica y la llevaron a Túnez y Berbería"30.

\section{DOCUMENTACIÓN INÉDITA SOBRE LOS ANDALUSÍES Y LA CHECHÍA, EN EL SIGLO XIX}

La bibliografía y la documentación sobre la industria de la chechía en el siglo XIX es muy abundante y está bien presentada en la bibliografía ya mencionada. Está relacionada con otros comercios, especialmente de la lana, tanto de importación, por la especificidad de las lanas españolas para la fa-

\footnotetext{
26. Ver Ignacio BaUer LandaUer, Fr. Francisco Ximénez. Colonia Trinitaria de Túnez, Tetuán, 1934.

27. Estas informaciones han sido recogidas como apéndice: de M. de EPALZA, «Nuevos documentos...», pp. 224-228.

28. I. BAUER LANDAUER, op. cit., pp. 48-49.

29. Ibídem, p. 27.

30. Ibídem, p. 40.
} 
bricación de las chechías, como por la producción local, que dependía de una administración estatal especializada ${ }^{31}$.

Ya estudiamos, en un artículo general, con mucha documentación inédita, española y tunecina, el conjunto de las relaciones entre España y Tunicia, también en lo relacionado con la chechía y el comercio de los productos relacionados con esta artesanía, a lo largo del siglo XIX ${ }^{32}$. Las relaciones comerciales hispano-tunecinas relacionadas con la lana española necesaria para la fabricación de la chechía tunecina, pueden resumirse, según la documentación del siglo XIX, en el párrafo siguiente:

"Quant aux activités commerciales, nous savons que la laine servant à fabriquer la «chéchia» venait traditionnellement d'Espagne. Mais elle transitait généralement par les ports français et italiens. Or, on doit probablement aux Soler l'établissement de relations commerciales directes entre l'Espagne et la Tunisie, car c'est Soler qui suggère qu'on payât les dettes du Bey en laine et que les Bonapartistes comme les insurrectionnels prissent garde qu'aucun dommage ne fût fait au représentant commercial du Bey et du ó_iib aô-ôaba' à Alicante, Luis Gianno. En échange de la laine, les Tunisiens envoyaient en Espagne du blé, de 1 'avoine et des féves" 33 .

Un informe consular de mediados de siglo, del nuevo cónsul español, en 1862, Eduardo Romea, escribe, en el apartado comercial: "Néanmoins, on reçoit indirectement, par les ports franca de Marseille, Gènes et Livourne, des laines de Ségovie et de Léon, qu'on emploie à la fabrication des bérets maures; il est interdit d'en fabriquer avec de la laine d'autres pays et d'autres qualités. On peut calculer qu'on en utilise de nos jours 1.000 kilos par an.

31. Ver Heger MAZOuz BEN-ACHOUR, «Implantation andalouse et structuration du Dār al-jild. Hypothèses sur les origines de cette ferme», Sharq al-Ándalus. Estudios Árabes, 7, Alicante, 1990, pp. 11-14. El Där al-ŷild o "Casa de la Piel" era un monopolio del Estado, "encargado de la recolección y de la comercialización de los cueros, de la cera y de la lana en la Regencia de Túnez", desde finales del siglo XVII a finales del XIX. Sus funciones económicas estaban arrendadas ("ferme") a ricos comerciantes, generalmente judíos, y su implantación corresponde a regiones próximas a la capital, donde estaban instalados pueblos colonizados por los moriscos y sus descendientes andalusíes desde el siglo XVII. En esos pueblos se realizaban también algunos de los procesos de la fabricación de los bonetes de lana o chechías. Ya se ha visto el caso de El Batán, junto a Tebourba.

32. M. de EPALZA y A.-H. EL GAFSI, «Relations tuniso-espagnoles au XIXe siècle: documenta et synthèses», Les Cahiers de Tunisie, XXVI, Túnez, 101-102, 1978, pp. 183-216. Sobre "les achats constante de laine espagnole, qui se font par Marseille, Génes ou Livourne” (p. 188), ver bibliografía en L. VALENSI, «Islam et capitalisme: production et commerce des chéchia en Tunisie et en France aux XVIIe et XIXe siècles», Revue d'Histoire Moderne et Contemporaine, París, 1969, pp. 389-390, y Le Maghreb avant la prise d'Alger 1790-1830, París, Ed. Flammarion, pp. 53-57.

33. M. de EPALZA y A.-H. El GAFSI, «Relations...», p. 199. 
On reçoit aussi des quantités considérables de sucres de La Havane, blanc et terciado, de la cochenille des Canaries, du plomb, du mercure, du safran et d'autres articles de moindre importante...".

El cónsul Malagamba no era más optimista en 1855. Precisaba:

"Le commerce indirect a été le même que les annés précédentes. Cependant on a pu remarquer une légérè augmentation de la laine lavée, car la situation des fabriquants de bonnets s'est amélioré et on constate une plus grande utilisation de ceux-ci dans le pays" 34 .

\section{LA DECADENCIA DE LA INDUSTRIA ARTESANAL DE LA CHECHÍA}

La artesanía de la chechía (o "bonete tunecino", según Jovellanos ${ }^{35}$-producto por excelencia de la artesanía y del comercio tunecinos-) era la actividad económica de exportación más importante del país, hasta las últimas décadas del siglo XIX (recuérdese que la ocupación colonial francesa, con el Protectorado, se inicia en 1881, pero la decadencia de esta industria artesanal no está directamente ligada con esta colonización, como se verá a continuación).

Aunque esta industria artesanal estaba instalada posiblemente en el país antes del siglo XVII, es con la llegada masiva de los moriscos expulsados de España, entre los años 1609 y 1614, cuando conoció una mutación profunda, particularmente en la época de Yúsef Dey (1610-1637), probablemente después de 1620. Los indicios de esta mutación son la construcción de un nuevo zoco, ya bajo el gobierno de Othmán Dey (1594-1610), la escasez de obreros y artesanos cualificados en la época anterior y el envío de lanas españolas por los puertos de Génova y de Liorna (Livorno) $)^{36}$.

La crisis de esta artesanía sólo se manifestará dos siglos más tarde, hacia 1830 Se debía, principalmente, a las perturbaciones de las relaciones de Tunicia con Levante y los territorios árabes bajo el poder otomano, por la guerra de Grecia y el corso griego, y por la competencia cada vez más importante de los bonetes de imitación, fabricados en Europa y particularmente en Francia. También pesaron, en esta evolución, el encarecimiento de las materias primas utilizadas en Tunicia (lana española y productos pictóricos

34. Ibídem, p. 304.

35. Ver R. RICARD, op. cit., y texto reproducido a continuación, en el Apéndice documental de este trabajo.

36. Ver Sadok BoubAKER, La Régence de Tunis au XVVIe siècle: ses relations commerciales avec les ports de l'Europe méditerranéenne, Zaghouan, Ed. C.E.R.O.M.D.I., 1987, p. 134. 
de Canarias), así como el endeudamiento de los boneteros ante los prestadores europeos ${ }^{37}$.

En 1837, la Regencia de Túnez continúa exportando la chechía a Argelia, a Tripolitania, a Egipto y a los Balcanes ${ }^{38}$, pero la reducción de las exportaciones de este precioso producto artesanal se hace sentir cada vez más y su parte en las exportaciones ya no representa más que el 12,6 o el $14,4 \%$ del total $^{39}$.

El declive de la exportación provoca la afluencia masiva de la chechía manufacturada francesa de imitación, a pesar de su calidad inferior. Es consecuencia directa de la revolución industrial y de la expansión colonial europeas. La chechía francesa se vende tanto en Tunicia como en otros países, con precios que no admiten competencia, como lo muestra esta tabla de 1890:

\begin{tabular}{lll}
\hline & CHECHIA TUNECINA & CHECHÍA EUROPEA \\
\hline 1890 & ordinaria: 6 francos & 1 a 1,50 francos \\
& de lujo: 7 a 8 francos & \\
1896 & 4 a 8 francos & 2,5 francos \\
1900 & 4 a 8 francos & 1,40 a 2,80 francos $^{40}$ \\
\hline
\end{tabular}
$1875^{41}$.

La disminución de la demanda tunecina se hace sentir ya muy sensiblemente a partir del año

Este declive se reforzó, evidentemente, por la aplicación de tratados muy perjudiciales para los intereses tunecinos, impuestos a los soberanos tunecinos por las potencias europeas, especialmente el régimen aduanero instituido el 21 de mayo de 1824, que cargaba sobre las exportaciones de la chechía tunecina con tasas que variaban entre el 8 y el $25 \%{ }^{42}$.

La instalación del Protectorado francés en Tunicia, a partir de la firma del Tratado del Bardo del 12 de mayo de 1881, la imposición de Francia sobre los circuitos económicos y políticos de la Regencia ${ }^{43}$ y, sobre todo, la

37. Ver Muhammad Hedi CHERIF, «Expansion européenne et difficultés tunisiennes de 1815 à 1830», Annales. Économies, Sociétés, Civilisations, 3, París, 1970, p. 731.

38. Ver Ahmad Kassab, Histoire de la Tunisie. L'époque contemporaine, Túnez, Ed. Société Tunisienne de Diffusion, 1976, p. 143.

39. Ver M.H. CHERIF, «Expansion...», pp. 727-728.

40. Ver Muhammad A. BEN ACHOUR, Catégories de la société tunisoise dans la 2ème moitié du XIXe siècle, Tunis, Ed. Institut National d'Archéologie et d'Art, 1989, p. 285.

41. Ver A. KASSAB, Histoire..., p. 145.

42. Ibidem, p. 135.

43. Ver M. de EPALZA y A.-H. El GAFSI «Relations...», p. 209. 
aplicación del nuevo régimen aduanero establecido en 1898 aceleraron profundamente ese proceso de declive iniciado medio siglo antes.

El contacto con el modo de vida europeo, acelerado a partir de 1881, tuvo como consecuencia inmediata el abandono progresivo del traje tradicional tunecino y, sobre todo, la renuncia a llevar la chechía. El vestirse a la europea se convirtió, para los tunecinos de religión musulmana y sobre todo de religión judía, en una forma ideal de adhesión a una forma de civilización dominante y en un trampolín de promoción social e intelectual; en definitiva, como una forma de resurrección o de renacimiento.

La gran crisis mundial de 1929-1934, con sus dos fenómenos económicos máximos de superproducción y de caída generalizada de los precios, conmovió profundamente toda la economía de la Regencia y particularmente la artesanía de la chechía.

Pero de todas formas, y a pesar de esas vicisitudes, mutaciones transformaciones y crisis, vividas, conocidas y sufridas por los tunecinos, que conmovieron profundamente las estructuras seculares de estas producción, la chechía tunecina resistió, se mantuvo, no desapareció, sobrevive y parece conocer actualmente una cierta recuperación de actividad, como consecuencia de la afluencia cada vez mayor de turistas de diversas partes del mundo y de la recuperación del traje tradicional con este tocado por parte de los tunecinos.

Así se han vuelto a instalar, en la actualidad, nuevas tiendas que fabrican chechías cara al público y las almacenan en la trastienda, en el zoco (souk) de los fabricantes de chechía ( $s \bar{q} q$ $a \check{s}-\check{s} a w a \tilde{s}(\overline{1})$, que ya ha recuperado parte de las apariencias de su antigua prosperidad, de hace siglos.

La disminución de las exportaciones (consecuencia de la competencia de los productos europeos, de cambios en las modas vestimentarias, etc.) y la reducción del mercado interior y exterior obligaron a un gran número de artesanos boneteros a reducir considerablemente su actividad. La disminución progresiva de las ganancias que siguió a esta evolución llevó a una degradación constante del nivel de vida, al debilitamiento del patrimonio familiar, al endeudamiento, al empobrecimiento... ${ }^{44}$. Las bases sociales y económicas de los artesanos boneteros se hallaron, por tanto, fuertemente resquebrajadas por la decadencia de las actividades de esta artesanía.

Esta larga y profunda crisis hacía también aleatoria la carrera en esta artesanía de la chechía. Hacia 1890 la artesanía de la chechía abarcaba unos 500 miembros, que hacia 1900 ya no eran más que $110^{45}$. Este ejemplo ilustra la disminución y la

44. Ver M.A. Ben Achour, Catégories..., p. 308.

45. Ver A. KASSAB, Histoire..., p. 150. 
caída de los efectivos de los miembros de esta artesanía. Esta evolución tuvo como consecuencia la emigración cada vez más fuerte de los compañeros y de los aprendices hacia los sectores de actividad modernos (talleres de tejidos mecánicos...) y la no renovación de los efectivos por gente más joven, que ya no estaban atraídos por una profesión en plena crisis.

El abandono de las tiendas a los compañeros y aprendices, la emigración de los maestros artesanos hacia la administración, hacia la enseñanza o hacia la explotación de las tierras familiares permitieron la intrusión de nuevos efectivos en esta artesanía y, sobre todo, la alteración de la homogeneidad profesional anterior. Eso produjo, consecuentemente, la degradación de las técnicas tradicionales de la corporación y muchos efectos de degeneración, de desarticulación y de disgregación, como resultado del abandono de los reglamentos de la corporación y de la liberación de las obligaciones corporativas que garantizaban a un tiempo la calidad del producto y su precio. A pesar de las tentativas de reorganización de la profesión, después de la promulgación del decreto del 12 de marzo de 1884, esta artesanía no conoció ninguna mejora.

Estas diferentes mutaciones, y, sobre todo, esta crisis profunda, engendraron, evidentemente, el desarrollo del movimiento sindical en el seno de la corporación de los fabricantes de chechía ( $\check{s} a w a \check{s} \tilde{1})$. Efectivamente, la huelga de 1924, que tocó diversos sectores económicos del país, tuvo como consecuencia la detención de varios responsables sindicalistas. Mohamed al-Kabadi, secretario general de los fabricantes de la chechía, figuraba en la lista de los inculpados ${ }^{46}$.

No es más que después de la legalización del movimiento sindical, a consecuencia de la promulgación del decreto del 16 de noviembre de 1932, cuando esta mutación empezó a manifestarse. Se tradujo en particular por el crecimiento del número de los artesanos sindicados (400 afiliados) y, sobre todo, por la escisión definitiva entre maestros artesanos ('aräf) y los obreros boneteros (compañeros - ashab- y aprendices -qalfa-). A partir de este momento, los compañeros y aprendices van a oponerse a sus amos o maestros artesanos ${ }^{47}$.

\section{PRESENCIA DE LOS LAKHOUA EN LA CORPORACIÓN DE LOS BONETEROS}

La presencia de los Lakhoua en la corporación de los boneteros era más que secular.

46. Ibidem, p. 400.

47. Ibídem, p. 161. 
La primera mención disponible, según sabemos, indica que la familia Lakhoua fue a menudo mencionada en compañía de otras familias de origen andalusí, como los Castellii ${ }^{48}$, Cortoby Ben Ashour, Jefine, en los cuadernos de los mercaderes marselleses Sabain y Beranger, entre los años 1696 y $1700^{49}$.

Como ya hemos expuesto, un cuarto de siglo más tarde el registro n. ${ }^{\circ} 2.249$ bis, de 1727/1140, depositado en los Archivos Nacionales Tunecinos, nos proporciona indicaciones preciosas sobre las tiendas de los boneteros en los zocos de los fabricantes de chechía. Según estas informaciones, cuatro miembros de la familia Lakhoua, probablemente de padres diferentes, ostentaban la propiedad de cuatro boticas, de un total de 18 . Eso representaba el $23 \%$, o sea casi la cuarta parte de las boticas censadas ${ }^{50}$.

Este mismo número está confirmado por el estudio hecho por L. Valensi, quien afirma que en los primeros años del siglo XIX se encuentran cuatro miembros de la familia en la corporación ${ }^{51}$.

La misma autora confirma también que "los Lakhoua forman parte de las tres familias que se destacan en el conjunto, porque son las más antiguas en el oficio, las más activas y las más numerosas: los Lakhoua, Louzir y Sida. Las tres son de origen andalusí reconocido" ${ }^{2}$.

En 1862, una crisis grave afecta a las estructuras de la corporación, como consecuencia de las transacciones del amín o síndico. El maestro bonetero Lakhoua y otros dos maestros son los que encabezan las protestas. Efectivamente, este grupo dirige al Bey o soberano de Tunicia una petición, denunciando al mismo tiempo el favoritismo del amín y el no respeto de los reglamentos de la profesión ${ }^{53}$.

En 1880, había por lo menos ocho miembros de la familia en la corporación ${ }^{54}$. En 1884 ,

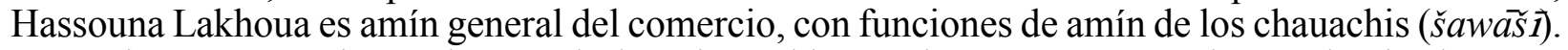
Este mismo personaje es el amín de los chauachis, productores y comerciantes de chechías, en $1885^{55}$.

Hamida Lakhoua, síndico de la corporación, que tenía la función de árbitro y experto de la profesión, reclamaba el 13 de noviembre de 1888 una indemnización de 60 francos y 15 libras de jabón por la custodia de los batanes

48. Ver A.-H. GAFSI, «Une famille de monétaires...», pp. 23-40.

49. Ver. S. BOUBAKER, op. cit., p. 165.

50. Ver A.-H. EL GAFSI, «Nazra...», p. 605.

51. L. VALENSI, «Islam et capitalisme...» p. 385.

52. Traducción del francés, de Lucette VALENSI, ibídem, p. 388.

53. Ver M.A. Ben ACHOur, Catégories..., p. 382.

54. Ibidem, p. 172.

55. Ibidem, pp. 172 y 397, nota 192. 
del río Medjerda, en El Bathan. Esta acción fue la primera manifestación de los boneteros contra la autoridad colonial, desde el establecimiento del Protectorado, en $1881^{56}$.

Los Lakhoua ocuparán una posición importante en el seno de la corporación, pero no parece que acapararan la función de amines de los chauachis ${ }^{57}$.

Después de la independencia de Tunicia, en 1955, varios boneteros dejaron la profesión para convertirse en funcionarios. Desgraciadamente, no tenemos indicaciones precisas y documentadas sobre este aspecto de la cuestión.

Las raras indicaciones disponibles nos permiten indicar que hasta nuestros días hay miembros de esta familia que prosiguen, a pesar de las dificultades de la profesión, en la práctica de este oficio. Slimane Lakhoua fue el informador de Teyssier, cuando este investigador hispanista francés realizó su encuesta sobre la chechía, hacia $1960^{58}$.

Este maestro-artesano bonetero era probablemente una de las figuras más importantes en este oficio, en el momento de esa encuesta. Efectivamente, según el encuestador, conocía perfectamente el vocabulario empleado en la confección de la chechía y, sobre todo, era consciente del origen español de muchas de las palabras empleadas ${ }^{59}$.

El abandono progresivo de la corporación, iniciado desde 1950, empujó a algunos de los jóvenes de la familia a emprender estudios superiores de medicina y de derecho en Francia. Otros prosiguen estudios en la facultad religiosa de la Zituna y siguen así fieles a cierta tradición familiar ${ }^{60}$.

La tabla n. ${ }^{\circ} 1$, realizada partiendo de un sondeo en los anuarios telefónicos de 1982, 1988 y 1994, indica esta nueva tendencia.

La misma tabla indica también que la Medina o barrio antiguo de Túnez ya no es el lugar de residencia preferido de la familia Lakhoua. Los nuevos barrios escogidos se sitúan en la "ciudad europea" o (al-baläd as-sīrì),

56. Ver S. FERCHIOU, Technique..., p. 165.

57. M.A. BEN ACHOUR, «Les Aylat...», p. 129.

58. «Le vocabulaire d'origine espagnole dans l'industrie tunisienne de la chéchia», Mélanges offerts a Marcel Bataillon par les hispanistes français, en Bulletin Hispanique, LXIV bis, Bordeaux, 1962, pp. 732-740, reproducido y presentado en M. de EPALZA y R. PETIT, Recueil d'études..., pp. 308-316.

59. Ver op. cit., con menciones generales y específicas del Sr. Slimane Lakhoua, pp. 309-310: «Notre source principale d'information a été M. Slimane Lakhoua, qui appartient à une famille de maîtres "chaouachis" dont les ancêtres sont venu autrefois d'Espagne. M. Lakhoua, qui est âgé de soixante-cinq ans, connaît le vocabulaire de la profession et est conscient de l'origine espagnole de beaucoup de mots qu'il cite», y p. 313, "notre informateur principal".

60. A. KASSAB, Histoire..., p. 130. 
que se suele oponer tradicionalmente a la "ciudad árabe" (al-balad al- 'arab_). Se encuentra a miembros de esta familia en las calles de España, de Montfleury, etc., o en chalets de las afueras: Ariana, Menzah, Khaznadar...

Estas nuevas elites del país han realizado probablemente sus estudios de enseñanza media en el colegio Sadikí, fundado en 1875 por el gran ministro reformador, el general Kheireddine. Eso indica la tabla siguiente ${ }^{61}$.

\section{Lista de los alumnos descendientes de la familia Lakhoua inscritos en el colegio Sadikí entre} 1875 y 1953

\begin{tabular}{llll}
\hline AÑOS & NOMBRES & AÑOS & NOMBRES \\
\hline 1880 & Tahar & 1943 & Slaheddine \\
1883 & Hedi, Mohamed & 1945 & Hammouda \\
1889 & Abdelaziz & 1950 & Jalel, Mohamed \\
1936 & Mohamed & 1951 & Mohamed \\
1942 & Mohamed, Mohsen & 1953 & Noureddine \\
\hline
\end{tabular}

\section{LOS LAKHOUA EN LA ADMINISTRACIÓN}

Los sondeos realizados en la Ruznāma y en At-takwìm at-Tūnisī dos almanaques tunecinos, indican que varios miembros de esta familia ilustre habían abrazado la carrera administrativa.

\begin{tabular}{|c|c|c|}
\hline NOMBRE & PUESTOS O CARGOS ADMINISTRATIVOS & FUENTE \\
\hline$\overline{\text { Ahmed }}$ & Califa en Mahdia en 1901 & Ruznāma, 1319/1901, pp. 293,381 \\
\hline “ & Gobernador en Bousalem en 1909 & Ruznāma, 1327/1909-1910, p. 211 \\
\hline “6 & Gobernador en Zaghouan en 1922 & At-takwīm, 1341/1922-1923, p. 114 \\
\hline Mohamed & $\begin{array}{l}\text { Funcionario en el Ministerio de } \\
\text { Justicia en } 1901,1909\end{array}$ & $\begin{array}{l}\text { Ruznäma, } 1319 / 1901-1902, \text { p. } 152, \\
\text { y } 1327 / 1909-1910, \text { p. } 113\end{array}$ \\
\hline Salah & $\begin{array}{l}\text { Intérprete en la Conservación } \\
\text { Inmobiliaria (defter-jana) en } 1925\end{array}$ & At-takwīm, 1344/1925, p. 228 \\
\hline Bechir & $\begin{array}{l}\text { Funcionario en el Ministerio de } \\
\text { los Bienes Inalienables (ýami' at } \\
\text { al-alhbās) en } 1922\end{array}$ & Ruznāma, 1341/1922-1923, pp. 69-70 \\
\hline Khemais & $\begin{array}{l}\text { Funcionario en el Ministerio de } \\
\text { la Guerra en } 1922\end{array}$ & At-takwīm, 1341/1922-1923, p. 88 \\
\hline Alí & $\begin{array}{l}\text { Funcionario en el Ministerio del } \\
\text { Trabajo en } 1902\end{array}$ & Ruznāma, 1320/1902-1903, p. 338 \\
\hline
\end{tabular}

61. A. AbDessalam, Sadiki et les sadikiens, Tunis, Céres Productions, 1975, p. 188 y ss. 
En puestos de agentes de ejecución más que directivos, generalmente, estos funcionarios muy raras veces acceden a puestos de responsabilidad, ocupados casi siempre por franceses.

Varios miembros de la familia se consagran a la enseñanza en la prestigiosa universidad religiosa de la Zituna. Entre las dos guerras mundiales, se encuentran allí dos enseñantes: Belhassen y Mohamed ${ }^{62}$.

La familia contaba también con varios notarios (' $u d \bar{u} l)^{63}$.

Pero el miembro más distinguido de la familia Lakhoua, en el siglo XX, ha sido sin duda el famoso Primer Ministro (al-wazĩr al-akbar) Hedi Lakhoua. Éste siguió una brillante carrera administrativa, coronada por el acceso al puesto de Primer Ministro, de 1932 a $1942^{64}$, después de haber ocupado el puesto de Ministro del Cálamo (wazĩr al-kalam, Ministre de la Plume), en los años treinta. Este último puesto le permitía ya asumir el ínterin del Primer Ministro, en caso de ausencia de éste, de leer los discursos del Bey en ciertas circunstancias, etcétera ${ }^{65}$.

Durante el período en el que estuvo en el Gobierno de la Regencia de Túnez, bajo el Protectorado francés, Hedi Lakhoua tuvo que afrontar dos acontecimientos importantes en la historia de Tunicia entre las dos guerras mundiales.

El primero fue la celebración del Congreso Eucarístico Internacional, en Cartago, el mes de mayo de 1930. Este Congreso Eucarístico fue sentido como una ofensa de Francia contra el Islam y contra los tunecinos. Hedi Lakhoua, como Ministro del Cálamo, recibió las llamadas a la protesta, en forma de peticiones firmadas que provenían de los nacionalistas tunecinos ${ }^{66}$.

El segundo se relacionaba con la fatwa (consulta jurídica religiosa) emitida por el tribunal religioso de la Charaa ( $\check{s} a r \bar{i} a^{\prime} a$ ) para legitimar la inhumación de los tunecinos que habían tomado la nacionalidad francesa ("naturalisés français"), en los cementerios musulmanes.

Hedi Lakhoua, como Primer Ministro, estuvo implicado en la elaboración de este procedimiento jurídico y de este acto político-religioso ${ }^{67}$.

Este asunto provocó un descontento popular, seguido de incidentes y de manifestaciones en todo el país. La finalidad de estos actos era evidente-

62. Información oral de Mongi Neifar, investigador en el Instituto Nacional del Patrimonio, del Ministerio de Cultura tunecino.

63. M.A. Ben Achour, Catégories..., p. 172.

64. Ídem, «Les Aylat...», p. 130, y Catégories..., p. 173.

65. A. MAHJOUBI, Les origines du mouvement national en Tunisie 1904-1934, Tunis, Faculté de Lettres, 1982, p. 471; A. KASSAB, Histoire..., p. 281.

66. A. MAHJOUBI, Les origines..., p. 471.

67. Ibidem, p. 489. 
mente el oponerse al entierro en los cementerios musulmanes de tunecinos "naturalizados" franceses ${ }^{68}$.

A pesar de estos dos episodios poco gloriosos en su carrera administrativa y política, Hedi Lakhoua fue objeto, entre otros, de un panegírico en verso escrito por el gran erudito tunecino Mohamed Mokdad El Wartalani. Esta obra fue escrita en 1932, con ocasión de la descripción de la gran mezquita de Testour, probablemente después de su restauración. El autor hace un remedo de la muwaššaha de Ibn Sahl de Sevilla ${ }^{69}$.

Antes de ser nombrado Ministro del Cálamo, Hedi Lakhoua había ocupado cargos administrativos importantes como primer intérprete en el Consejo Municipal de la capital (al-ma ŷlis al-baladì), en $1902^{70}$ y probablemente hasta $1909^{71}$ y más aún. Pasó después al Ministerio del Cálamo, donde se le encuentra como funcionario en el servicio de contabilidad, en $1916^{72}$. En 1922, es funcionario en el ayuntamiento de la capital (mašjat al-madina $)^{73}$.

Por otra parte formó parte de varias asociaciones de carácter cultural y nacionalista. Así se le encuentra en 1897 como miembro de la Association Khaldounienne (al ŷam iyya al-jaldüniyya) sólo al año de haberse fundado ${ }^{74}$. En 1906, Hedi Lakhoua es miembro de la Asociación de los Antiguos Alumnos del Colegio Sadikí (Association des Anciens du Collège Sadiki), fundada en $1905^{75}$.

Sus hijos ocuparon diversas dignidades en la corte del Bey, reforzando así la afición de los miembros de la familia por la carrera administrativa, afición iniciada por su abuelo Mahmoud, que fue secretario en la Sección de Estado o Primer Ministro, y por su tío Ahmad, que fue intérprete y luego alcaide $\left(q \bar{a}^{-} \mathrm{i} i d\right)$ o gobernador ${ }^{76}$.

FORTUNA INMOBILIARIA DE LA FAMILIA

Como ya se ha visto, la familia Lakhoua disponía de una fortuna inmobiliaria considerable, adquirida esencialmente gracias al comercio de los bo-

68. Ibídem, p. 497.

69. A. Hamrouni, Testour. Histoire et récits de voyage, Túnez, Mediacom, 1995, p. 35.

70. Ruznāma, 1902-1903/1320, p. 299.

71. Ruznāma, 1909-1910/1327, p. 162.

72. Ruznāma, 1916-1917/1335, p. 137.

73. Ruznāma, 1922/1341, p. 68.

74. Ver M. SAYADI, Al jamiyya al khalduniyya 1896-1958, Tunis, Maison Tunisienne de 1’Édition, 1974, p. 50.

75. Ver Ruznāma, 1906-1907/1324, p. 354.

76. M.A. BEN ACHOUR, «Les Aylat...», p. 130. 
netes ${ }^{77}$. Importaba diversos artículos de calidad (tejidos, especies, maderas, perfumes apreciados en Túnez... $)^{78}$. Esta fortuna le había permitido poseer muchas propiedades inmobiliarias constituidas por tierras cerealícolas (henchires), olivares y vergeles en zonas cercanas a Túnez capital (Ariana, Mornag, Zaghouan... $)^{79}$. Esta fortuna le había permitido poseer también amplias mansiones en los principales barrios de la Medina de Túnez (calle de los Andalusíes, calle Sidi Surdu ${ }^{80}$, calle del Pachá...) y especialmente en el palacio de Dar Lakhoua, en la calle de los Andalusíes (nahj Al-Andalus ${ }^{81}$.

Como casi todas las mansiones burguesas de la Medina de Túnez, esta "dâr" se distingue por

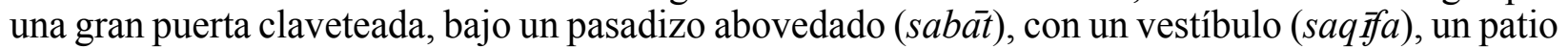
con cielo abierto, pozo, cisterna y un estanque. Esta casa da a la calle gracias a unos amplios ventanales, cubiertos con celosías (mušarabiyya). Pero las mansiones de esta familia se distinguen particularmente por tener unas buhardillas o depósitos (majzin) destinados a guardar las lanas, los bonetes y los talleres familiares.

Esta mansión, en particular, se distingue por una fachada sencilla dominada por una bellísima puerta claveteada, con aldabas. Esta puerta da acceso a un vestíbulo (saq îfa) cubierto, con losas calcáreas y con bóvedas de ladrillo visto, paredes con bonitos azulejos policromados y con frisos de arcos de estucos cincelados (naqša hadīda), de estilo andalusí. El piso superior tiene un salón que da al mismo tiempo al patio y al exterior. Tiene también varias habitaciones, que se utilizaban antes para talleres familiares.

Junto a esta casa y en la misma fachada se encuentra una segunda que se distingue por tener cinco vestíbulos (saqä'if) y un patio con otro patio pequeño adjunto, con linterna central, con habitaciones para los huéspedes.

La tercera y cuarta "Casa Lakhoua" (darr al-ijwa) se encuentran situadas en la mitad de la calle de los Andalusíes (nahŷ al-andalus, "rue des Andalous"). Las dos casas obtienen luz por el intervalo que separa a dos pasadizos abovedados. Dos puertas con arcos, de madera claveteada, decoran la fachada.

77. Ibídem.

78. Ver M.A. Ben AHour, Catégories..., p. 293.

79. Ibidem, p. 262.

80. Sobre este personaje morisco, de apellido hispano ("el Señor Alí El Sordo Jalaf ad-Dūn", italianizado "Calafatino"), ver documentación en M. de EPALZA, «Moriscos y andalusíes...», pp. 247-327 (especialmente pp. 269 y 298-299), traducido al francés en M. de EPALZA y R. PETIT, op. cit., pp. 150-188, con fotografía de su tumba, p. 187, conservada en la casa n. ${ }^{\circ} 16$ de la calle. La documentación abarca desde 1654 a 1659 , en que el rico comerciante tunecino era representante del gobernador genovés de Tabarca para el rescate de esclavos (él mismo había sido apresado por un francés, en 1653, en uno de sus viajes comerciales, entre Túnez y Liorna -Livorno-).

81. Ver descripción en J. REVAult, Palais..., especialmente t. II, p. 150, sobre la Dār Lakhoua de la calle Sidi Es-Sourdou, texto reproducido en M. de EPALZA y R. PETIT, op. cit., p. 302. 
Una da acceso al piso de los huéspedes y la otra permite la entrada a la cuarta mansión. Una tienda situada frente a la entrada servía como depósito de jarras de aceite y otras provisiones que se traían de las propiedades agrícolas de los Lakhoua situadas en la región de Ariana, Mornag y Zaghouan.

El piso de los huéspedes está construido alrededor de un patio con suelo de mármol, adornado con una cornisa de madera pintada sobre columnas y con paneles de azulejos. Permite el acceso a tres hermosas salas de recepción ricamente amuebladas. Las paredes están cubiertas con azulejos y con paneles de estuco labrado.

La cuarta casa tiene casi la misma planta y lleva la misma decoración. La "Casa Lakhoua" de la calle Sidi Surdu tiene una fachada que se distingue por una gran puerta claveteada, en un arco de piedra esculpida con motivos geométricos y florales y debajo de una triple ventana saliente con reja de hierro forjado y celosía de madera pintada (barmakli), que forma una mucharabía. Esta puerta permite el acceso a un vestíbulo (driba) recubierto con bóvedas de ladrillos en forma de espinas, con un banco de pared, de madera (dukkāna), reservado para acoger a los visitantes. A ese vestíbulo dan unas puertas que van al piso superior, a los almacenes (majzin) que se utilizan para conservar las lanas destinadas a la confección de las chechías y al jardín (jnina).

Por este vestíbulo se accede a un patio a cielo abierto, dominado por dos pórticos simétricos adornados con un arco, con columnas, con capiteles con voluta y media luna, con decoración de estuco y de paneles de cerámica y con una cornisa de tejas. Este patio se abre a cuatro habitaciones con triple alcoba, ricamente adornadas, y a una cisterna y una cocina dotada de un pozo, de un baño (hammam) y con una habitación para las provisiones (bayt al-mūna). El piso superior está dominado por un patio cubierto por un lucernario iluminado por ventanas que dan a dos hermosas salas de recepción.

Esta casa está pegando, en su parte exterior, a una mezquita.

\section{CONCLUSIÓN}

Los Lakhoua se integran perfectamente en Túnez, por su origen social, por pertenecer a la «comunidad andalusí», por su competencia y éxito en la profesión de mercaderes de bonetes y por el papel que desempeñan en la economía del país y en la sociedad urbana de la capital. Consiguen ocupar un puesto importante en esa sociedad, imponerse en la ciudad de Túnez y beneficiarse, por tanto, de un gran prestigio. El hecho de que los Lakhoua pertenezcan a la "comunidad andalusí y al grupo socio-profesional de los chauachis se refleja en las alianzas matrimoniales entre familias de esa "comunidad", de ese grupo socio-profesional o de los notables del mundo de los zocos en general, los ambientes del poder político (majzin), de la ciencia y 
la cultura religiosa ( 'ilm) o de la devoción de cofradías (zāwiya). Estas alianzas hacen aún más sólida una integración en la sociedad receptora, con un profundo enraizamiento y múltiples formas de promoción social. Para estos andalusíes, la endogamia socio-profesional y la monogamia son generales, mientras que la endogamia biológica es muy reducida.

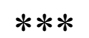

\section{APÉNDICE DOCUMENTAL ${ }^{82}$}

Este texto del político y escritor español Gaspar Melchor de Jovellanos (1744-1811), «Informe sobre un proyecto de fabricación de gorros tunecinos», va dirigido al Conde de Lerena, D. Pedro López de Lerena, Secretario de Estado [Ministro] de Hacienda (1734-1792) ${ }^{83}$. Sitúa la evolución de esta artesanía pre-industrial, la de la chechía o "gorro tunecino", antes y después de la expulsión de los moriscos ${ }^{84}$. Es un texto complementario de la investigación que acabamos de presentar.

82. Texto sacado de la edición de la Biblioteca de Autores Españoles, Obras publicadas e inéditas de Don Gaspar Melchor de Jovellanos, colección hecha e ilustrada por Don Cándido Nocedal, Madrid, Ediciones Atlas, 1952, t. II, pp. 64-66. Agradezco a la profesora Dra. Mercedes Sánchez, de la Universidad de Oviedo, el que me haya proporcionado copia del texto, por mediación del Dr. Míkel de Epalza, de la Universidad de Alicante, ejemplo de cooperación investigadora para hacer adelantar nuestro conocimiento sobre este campo de actividades de los moriscos y sus descendientes después de la expulsión de España y su instalación en Tunicia.

83. El texto fue remitido el 14 de junio de 1787 con la siguiente carta:

"Excelentísimo señor.- Muy señor mío: Dirijo a vuecencia el informe que se sirve pedirme por su papel del 13 del pasado, no habiéndolo despachado antes por esperar más noticias de Cataluña, que al cabo no han venido, como deseaba.

No me atrevo a indicar el cuánto de los auxilios que se pueden señalar a este fabricante. En este punto es aventurado todo cálculo que no se haga con un perfecto conocimiento del pormenor de estas manufacturas y fondos necesarios para ellos, y este conocimiento me falta del todo.

Por esto creo que será lo mejor informarse del cónsul de Marsella, puesto que en Cataluña esta manufactura es un accesorio de otras, y en Mallorca corren las operaciones por muchas y muy diversas manos.

Yo celebraré haber llenado los deseos de vuecencia; el mío es que me continúe sus órdenes mientras ruego a Nuestro Señor etc." (ibídem, p. 66, nota 1).

Sobre el escritor y economista Conde de Lerena [no Marqués, como creen algunos], que llegó a ser de hecho Secretario de Estado, cuando empezaba la decadencia del Conde de Floridablanca, ver Fabrice ABBAD y Didier OZANAM, Les intendants espagnols da XVlle siécle, Madrid, Casa de Velázquez, 1992, pp. 119-120, y Carlos de Amor, Quién es quién en la nobleza española?, Barcelona, Arce, 1985, p. 214. Político conservador, fue muy criticado por Jovellanos.

84. Este texto de Jovellanos llamó la atención del hispanista francés Robert RICARD, que lo presentó con algunas informaciones nuevas sobre el tema, en «L'Espagne et la fabrication des “bonnets tunisiens”. À propos d'un texte du XVIII siècle», Revue Africaine, Argel, tomo C, 1956, pp. 123-132. 
El texto parte de un hecho anecdótico: el ofrecimiento de un artesano francés al gobierno español. Es artesano fabricante de "bonetes tunecinos", producto equivalente en la época a la boina española, birrete toledano, llamado aún hoy en día en francés béret basque ("gorra vasca"). Consultado sobre el proyecto del francés, Jovellanos hace gala de erudición histórica y de afán de progreso socio-económico para la sociedad española, recomendando el proyecto, pero con medidas realistas, económica y administrativamente ${ }^{85}$.

"La proposición que con fecha 7 de marzo dirigió a vuecencia Juan Bertran, fabricante de bonetes o gorros tunecinos en Marsella, y que de orden de su majestad remite vuecencia a mi informe con su papel de 13 de abril anterior, se reduce a implorar de la generosidad de vuecencia los auxilios necesarios para establecer en España la misma manufactura.

Expone a este fin Bertran, que restablecida la paz con los berberiscos, puede pensar España en restaurar su antiguo comercio de bonetes; que el único vecino que puede competirle, la Francia, necesita para esta industria de nuestras lanas; que la falta o carencia de ellas obliga a los artistas franceses a viciar la materia de sus bonetes; que éstos sólo logran salida y despacho, porque la única fábrica de Túnez no puede abastecer las varias escalas de Levante, donde se consumen; que establecida esta industria en España, no podrá la de Francia sufrir su concurrencia ni conservarse; y que de aquí resultará la ruina de aquellas fábricas y la transmigración de sus obreros a las nuestras.

Ofrece en consecuencia Bertran al Ministerio de vuecencia los conocimientos adquiridos en los años de trabajo que tuvo en la fábrica de bonetes de Marsella, perteneciente a Juan Francisco Rozan; se manifiesta pronto a pasar a España con el objeto indicado; dice que su familia se compone de mujer, madre, una hermana, y otras cinco o seis personas; asegura que si tuviese fondos, sólo pretendería de vuecencia un permiso para establecerse acá; pero por falta de ellos los espera de su generosidad, y concluye sin poner condiciones ni pedir señaladamente cosa alguna.

El objeto de esta proposición merece la atención de vuecencia, pues aunque el uso de los gorros tunecinos se haya disminuido considerablemente, no hay duda que se puede hacer todavía un gran consumo de este género.

Fue esta manufactura muy celebrada entre nosotros por todo el siglo XVI; y lo era todavía en los principios del pasado, aunque ya entonces empezaba a lamentar su decadencia Damián de Olivares en sus escritos.

Había fábricas de bonetes en Sevilla, Córdoba, Granada, Valencia, Barcelona y Toledo, como prueban sus antiguas ordenanzas gremiales, siendo la de esta ciudad la más considerable de todas.

Si es cierto lo que asegura Francisco Martínez de la Mata en uno de sus discursos políticos, citado en el cuarto apéndice a la Educación popular, había por los años de 1624 en Toledo doscientos maestros boneteros, los cuales trabajaban cada uno dos cajones por semana; cada cajón contenía cuarenta docenas; por consiguiente trabajaban al año diez y nueve mil doscientos cajones; esto es, setecientas sesenta y ocho mil docenas.

85. Hemos modernizado la ortografía, sobre la del siglo XVIII de Jovellanos, ya puesta al día a fines del XIX, por el editor del texto, el escritor y político Cándido Nocedal (1821-1885), particularmente en lo que se refiere a la acentuación. 
Los bonetes tenían por aquellos tiempos, pero particularmente en el siglo XVI, gran consumo dentro de España, por ser entonces el cubierto ordinario de la gente del pueblo en todas nuestras provincias; pero su mayor consumo se hacía fuera del reino, en África y todo el Levante, donde los bonetes españoles tenían la primera estimación sobre los de Milán y Génova.

Varias causas concurrieron después a la decadencia de esta manufactura: primera, la carestía de los jornales, resultado del enorme aumento de dinero que atrajo a nuestra circulación el comercio de América, por lo cual ya a la mitad del siglo XVI sentían nuestras manufacturas la concurrencia con las extranjeras, como se infiere de una petición hecha a Carlos V por los procuradores de las Cortes de 1545; segunda, la expulsión de los moriscos verificada en 1610, en que salieron de España cerca de un millón de individuos, que eran por la mayor parte fabricantes y consumidores de esta manufactura; tercera, el uso de los sombreros, que se empezó a hacer general coetáneamente a esta época, siendo antes peculiar a la gente de distinción, que sólo los usaba para defenderse del sol, yendo de camino, y habiéndose usado después como cubierto común y ordinario desde la mitad del siglo XVII; cuarta, la interrupción de nuestro comercio de Levante por el corso de los berberiscos, que llegó al mayor extremo de insolencia por aquellos mismos tiempos, en que nosotros carecíamos ya de comercio activo y de marina mercantil, y aun de marineros para surtirlos, y de escuadras para protegerlos.

Estas causas acabaron enteramente con todas nuestras fábricas de bonetes, no subsistiendo en el día ninguna de las que en lo antiguo tuvieron tanto nombre.

Sin embargo, no es desconocida esta manufactura en España, pues se fabrican todavía bonetes o gorros tunecinos en Puigcerdá y Olot en Cataluña, sin que haya sido posible averiguar qué cantidad se trabajan.

Fabrícanse también en Mallorca, donde hacen estos bonetes a la aguja las mujeres del país, y acaban las demás operaciones hasta perfeccionarlos los individuos del gremio de boneteros, que se compone en Palma de veinte y cuatro maestros con catorce tiendas, como se ve en un estado de la industria de aquella isla, trabajado por su Sociedad patriótica, y publicado entre sus Memorias en 1784, al folio 251.

No sé que en otra alguna parte de España se fabrique esta manufactura, pues aunque en varias provincias del Norte se trabajaban gorros de varios gruesos, son por lo común de hilo o de algodón y no pertenecen al ramo de que hablamos.

El consumo de bonetes en España puede ser todavía considerable, pues los usan nuestros marineros, pescadores y gentes de mar, no sólo en las costas de Levante, sino también en las del Norte y Mediodía; y fuera de España se usan asimismo entre la gente de mar, particularmente en los puertos de África y Levante.

La lana, única materia de los bonetes o gorros tunecinos, la grana y añil, únicos ingredientes de su tinte, pues sólo se usan encarnados y azules, en una palabra, todo cuanto es necesario para la materia y forma de esta manufactura, abunda entre nosotros, son géneros propios nuestros o de nuestras colonias, y lo son exclusivamente.

No puede, pues, dudarse que será de gran importancia multiplicar estas fábricas en España, y lo será tanto más, cuanto es una manufactura vasta, fácil de aprender y ejecutar, en que pueden ocuparse mujeres, niños y otra porción de individuos que se vician en la ociosidad, y suelen perecer por falta de trabajo.

Acaso convendría establecer esta fábrica con preferencia en nuestra costa del Norte, ya para no perjudicar a las que hay hacia Levante, ya para surtir más cerca la marinería de aquella costa, ya para aprovechar la baratura de alimentos y jornales que hay en aquellas provincias, y ya, en fin, para dificultar el contrabando que pudiera hacerse con los bonetes de Túnez y Marsella. Galicia, Asturias y las montañas de Santander serían, a mi ver, las 
provincias más a propósito para situar esta industria. Como quiera que sea, resulta de lo dicho que si Bertran fuera capaz de cumplir lo que ofrece, se le debe juzgar acreedor a los auxilios que solicita del Gobierno.

Pero en la distribución de estos auxilios es necesario proceder con gran precaución y economía, no sea que el Gobierno desperdicie en este establecimiento, como en otros, gruesas cantidades, sin recoger el fruto deseado.

Y yo no opinaré jamás por la concesión de sueldos o salarios a estos artistas, pues sucede muy frecuentemente que en teniéndolos, cuidan más de disfrutarlos que de merecerlos. Tampoco por la oferta anticipada de pensiones y premios; porque al cabo se hace muy difícil negárselos, aun cuando no los merezcan, dándose muchas veces a la importunidad o a la compasión lo que no se debe a la justicia.

El mejor medio a mi juicio es dar generosamente auxilios para los nuevos establecimientos, franqueando anticipadamente los caudales necesarios para ellos, con la sola obligación de restituir el todo o parte, después de haberlos disfrutado y enriquecídose con ellos.

Este medio suele tener el inconveniente de que los artistas aventureros no hallen quien les fíe o abone, y sin otra precaución, suele ser con ellos muy arriesgada la generosidad.

Pero a este inconveniente se puede ocurrir de dos maneras, a saber, tomando conocimiento anticipado del sujeto que se protege, para que a lo menos responda por él la experiencia de su conducta, y dándole principalmente los auxilios en especie, para que no los pueda malbaratar, sino ponerlos a logro.

Procediendo sobre estos principios, me parece que a la proposición de Juan Bertran se puede resolver lo siguiente:

1. ${ }^{\circ}$ Que se indague por medio del cónsul de su majestad en Marsella quién es Bertran, si tiene los conocimientos, práctica y buen propósito que indica, y si en él concurren calidades que prometan el buen cumplimiento de lo que ofrece.

2. ${ }^{\circ}$ En caso de tenerlas, se le prometerá una decente ayuda de costa para venir a España y trasladar a ella su familia; debiendo hacer este viaje a su riesgo, sin que el Gobierno se comprometa en manera alguna a facilitarle la salida, a cuyo fin nada se le anticipará ni dará hasta después de haber llegado.

3. ${ }^{\circ}$ Que se ha de establecer la manufactura de bonetes en la provincia y pueblo que el Gobierno le señalare, no quedando a su arbitrio esta elección en manera alguna.

4. ${ }^{\circ}$ Que para establecer dicha manufactura se le darán, bajo de seguro abono, y por costo y costas, todas la máquinas, instrumentos, materias e ingredientes necesarios para el cardado, hilado, tejido, perchado, tinte, forma y prensa de los bonetes, gorros, medias abatanadas y demás géneros de su arte, como también el caudal que pareciere necesario para mantenerse en el primer año; todo bajo la obligación de restituirlo en la forma que después se dirá.

5. ${ }^{\circ}$ Que por cada telar que pusiere corriente y trabajare por espacio de un año a lo menos, se le abonará una cantidad determinada, la cual se irá rebajando del capital que importaren los auxilios que se le hubiesen anticipado, reduciendo a menos por este medio la obligación de restituirla.

6. ${ }^{\circ}$ Que por cada oficial español que diere completamente enseñado en todas las operaciones de su arte, a satisfacción del Gobierno, y de tal forma que sea capaz de establecer por sí y dirigir la misma manufactura, le abonará otra cantidad determinada.

7. ${ }^{\circ}$ Que se concederán a su fábrica todas la gracias y franquicias que logran las demás fábricas de lana del reino, y particularmente las de bonetes y medias de Cataluña. 
8. ${ }^{\circ}$ Que sin embargo de deberse entender prohibida la entrada de bonetes o gorros extranjeros en el reino, como comprendidos bajo el nombre de cosas hechas, de que habla la ley 52, título XVIII, libro $6^{\circ}$, de la Recopilación, se hará además particular declaración prohibiendo en forma específica la introducción de dichos géneros en nuestros puertos.

9. ${ }^{\circ}$ Que para el pago del resto de la cantidad que importare el principal de los auxilios anticipados, después de hechas las rebajas correspondientes, se le dará el plazo de seis años, dentro de los cuales deberá verificar su retribución sin remisión alguna.

$10 .^{\circ}$ Que si el éxito de esta empresa fuese favorable, y tal que el Gobierno experimente una considerable y cierta utilidad, se le conceda un premio proporcionado al tamaño del servicio que hubiese hecho, sin que pueda exigir que anticipadamente se le señale cantidad ni recompensa alguna determinada; debiendo esperar de la generosidad del Gobierno que, si desempeñase sus promesas, no dejará defraudadas sus justas esperanzas.

11. ${ }^{\circ}$ Que el señalamiento de la cantidad que se haya de ofrecer a Bertran, tanto por el viaje, manutención del primer año, como por la enseñanza de oficiales, se haga después de oído el cónsul de Marsella, el cual, teniendo consideración a la habilidad y prendas del sujeto, a los fondos necesarios para conducir esta manufactura, y a la utilidad que puede producir anualmente cada telar, propondrá al Gobierno las que le parecieren convenientes, distribuyéndolas de tal modo que en el citado plazo de seis años pueda Bertran con su aplicación y trabajo enjugar la mayor parte de los auxilios recibidos, y hacerse acreedor al residuo, que en el caso de buen cumplimiento, se le puede abonar por vía de única recompensa.

12. ${ }^{\circ}$ Que este establecimiento se ponga a su tiempo bajo la inspección de la Junta de Comercio y Moneda, a quien se encargue por su majestad la vigilancia sobre la conducta de Bertran, la ejecución de sus promesas, y la observancia de las condiciones con que se aceptare.

Vuecencia resolverá lo que fuere de su mayor agrado.-Madrid, 14 de junio de 1787.

\section{RESUMEN}

La familia tunecina Lakhoua, de origen morisco, procede de emigrantes del siglo XVII pero también de emigrantes de Granada del siglo XVIII. Desde el XVII se distinguen en la artesanía pre-industrial de los bonetes chechía, una de las primeras riquezas de exportación del país, con técnicas y vocabulario de origen hispánico, que les permitió acceder a una categoría social y económica relevante en Túnez y su región. Se estudia la documentación tunecina y la española (Francisco Ximénez, s. XVIII) sobre esta familia y la evolución social de la fabricación de la chechía, en los cuatro últimos siglos. En apéndice, reproducción del informe de Jovellanos al ministro Conde de Lerena (1787) sobre la historia y situación española e internacional contemporánea de la fabricación de los "bonetes tunecinos".

\section{ABSTRACT \\ The Lakhoua famify, Tunisian descendants of Moriscos from Granada of the XVII-XVIII century and their activities in the industry of the 'Chechfa' caps. The}


Tunisian family Lakhoua, of Morisco origin, comes from emigrants of the XVII century but also from others of Granada of the XVIII. From the XVII century they were distinguished for the pre-industrial craft of the 'chechía' caps. This was one of the first profitable resources exported by the country. The use of techniques and vocabulary of Hispanic origin raised them to an excellent social and economic category in Tunisia and its region. The Tunisian and Spanish documenta (Francisco Ximénez, s. XVIII) on this family and the social evolution of the production of the 'chechía' in the last four centuries are studied. In the Appendix, the report of Jovellanos to the Minister 'Conde de Lerena' (1787) on the history and the Spanish and international situation contemporaneous with the production of the 'Tunisian caps' is reproduced. 